\title{
Cost-effectiveness of preoperative motor mapping with navigated transcranial magnetic brain stimulation in patients with high-grade glioma
}

\author{
Vicki M. Butenschön, MD, MScHE, Sebastian Ille, MD, Nico Sollmann, MD, PhD, \\ Bernhard Meyer, MD, and Sandro M. Krieg, MD, MBA \\ Department of Neurosurgery, Klinikum rechts der Isar, Technische Universität München, Munich, Germany
}

OBJECTIVE Navigated transcranial magnetic stimulation (nTMS) is used to identify the motor cortex prior to surgery. Yet, there has, until now, been no published evidence on the economic impact of nTMS. This study aims to analyze the cost-effectiveness of nTMS, evaluating the incremental costs of nTMS motor mapping per additional quality-adjusted life year (QALY). By doing so, this study also provides a model allowing for future analysis of general cost-effectiveness of new neuro-oncological treatment options.

METHODS The authors used a microsimulation model based on their cohort population sampled for 1000 patients over the time horizon of 2 years. A health care provider perspective was used to assemble direct costs of total treatment. Transition probabilities and health utilities were based on published literature. Effects were stated in QALYs and established for health state subgroups.

RESULTS In all scenarios, preoperative mapping was considered cost-effective with a willingness-to-pay threshold < $3^{*}$ per capita GDP (gross domestic product). The incremental cost-effectiveness ratio (ICER) of nTMS versus no nTMS was 45,086 Euros/QALY. Sensitivity analyses showed robust results with a high impact of total treatment costs and utility of progression-free survival. Comparing the incremental costs caused by nTMS implementation only, the ICER decreased to 1967 Euros/QALY.

CONCLUSIONS Motor mapping prior to surgery provides a cost-effective tool to improve the clinical outcome and overall survival of high-grade glioma patients in a resource-limited setting. Moreover, the model used in this study can be used in the future to analyze new treatment options in neuro-oncology in terms of their general cost-effectiveness.

https://thejns.org/doi/abs/10.3171/2018.3.FOCUS1830

KEYWORDS cost-effectiveness; high-grade glioma; motor mapping; nTMS

$\mathrm{W}$ ORLD Health Organization (WHO) grade III and IV gliomas are malignant tumors of the brain and represent the majority of primary brain tumors developing from glial cells. Although no curative treatment is yet known, advances in neuro-oncological treatment nowadays include a multimodal concept of therapy: cytoreductive surgery followed by combined radiotherapy (RTx) and chemotherapy (CTx) using temozolomide (TMZ).
Many studies have found a significant correlation between the extent of resection (EOR) and the overall survival (OS) of patients with these tumors, and extensive resection remains, whenever possible, the gold standard. . $^{13,14,22}$ This standard approach can be enhanced by several preand intraoperative tools, such as neuronavigation, intraoperative neuromonitoring, 5-aminolevulinic acid (5-ALA), and motor/language mapping prior to surgical removal of eloquent tumors.

ABBREVIATIONS 5-ALA = 5-aminolevulinic acid; CEAC = cost-effectiveness acceptability curve; $\mathrm{CHOICE}=\mathrm{CHOosing} \mathrm{Interventions} \mathrm{that} \mathrm{are} \mathrm{Cost-Effective;} \mathrm{CR}=$ complete resection; CTx = chemotherapy; EOR = extent of resection; fMRI = functional magnetic resonance imaging; GBD = Global Burden of Disease Study; GDP = gross domestic product; GTR = gross-total resection; HGG = high-grade glioma; ICER = incremental cost-effectiveness ratio; MEG = magnetoencephalography; nTMS = navigated transcranial magnetic stimulation; $\mathrm{OS}=$ overall survival; $\mathrm{PFS}=$ progression-free survival; $\mathrm{PR}=$ partial resection; $\mathrm{PSA}=$ probabilistic sensitivity analysis; $\mathrm{QALY}=$ quality-adjusted life year; RTx = radiotherapy; TMZ = temozolomide; WHO = World Health Organization; WTP = willingness-to-pay; YLD = years lived with disability; YLL = years of life lost.

SUBMITTED January 15, 2018. ACCEPTED March 14, 2018.

INCLUDE WHEN CITING DOI: 10.3171/2018.3.FOCUS1830. 
The current gold standard for postresection treatment of patients with newly diagnosed high-grade gliomas (HGGs) includes adjuvant therapy with concomitant radiotherapy and chemotherapy with TMZ, followed by chemotherapy alone (Stupp protocol). Despite the extensive multimodal treatment, the mean survival is limited. One reason is the challenging identification of tumor margins in locations close to highly eloquent areas, which makes it difficult to achieve complete resection. ${ }^{21}$

In addition to imaging techniques such as functional magnetic resonance imaging (fMRI), navigated transcranial magnetic stimulation (nTMS) can be used to identify eloquent cortex noninvasively. It was even shown that preoperative nTMS improves the $\mathrm{EOR}^{23}$ and the clinical outcome. ${ }^{12,13}$ Based on the principle of electromagnetic induction, we can identify the cortical representation of the motor cortex by measuring motor evoked potentials from a specific area of the brain. Recent studies have shown that nTMS is superior to other noninvasive techniques, such as fMRI and magnetoencephalography (MEG). ${ }^{23}$ It was therefore recently reported to be the most reliable preoperative mapping option for neuro-oncological centers if providing state-of-the-art care..$^{20}$

Nowadays, health care providers and consumers benefit from scientific progress but also face multiple challenges, including a surplus of treatment options conflicting with evidence-based therapy regimens; a rapidly changing industry and patient population; and of course, increasing health care expenses and financial boundaries.

Only a few publications have considered the economic impact implied by the clinical benefits of alternative treatment options and optimizations. Published articles have analyzed the economic benefit of 5-ALA for intraoperative tumor resection and the financial outcome of carmustine implants as well as intraoperative neurophysiological monitoring. ${ }^{8,10,11,21}$ To our knowledge, no study has been conducted on the economic evaluation of nTMS for preoperative motor mapping. Thus, the aim of this study is to estimate the financial burden of disease and the costeffectiveness of nTMS for HGG patients.

This study also provides a model that can be used in the future to analyze any new treatment option in neurooncology in terms of its general cost-effectiveness.

\section{Methods \\ Patients}

We included cohort patients in our simulation model, based on adults older than 18 years with histologically diagnosed HGG (WHO grades III and IV) who underwent surgical and adjuvant therapy in Germany in our university hospital following standard clinical practice (Table 1). We simulated several clinical scenarios for the treatment option (with nTMS) and the comparator cohort (no nTMS):

1. EOR: gross-total resection (GTR) versus partial resection (PR)

2. Motor deficits (no vs present deficits)

3. At each stage, the corresponding probability of death was included

The probabilities of complete resection (CR) and PR
TABLE 1. Utility values

\begin{tabular}{lccc}
\hline \multicolumn{1}{c}{ Health State } & Value & Reference & $\begin{array}{c}\text { Variation } \\
\text { for PSA }\end{array}$ \\
\hline uProgressionFree_nodeficit & 0.8872 & Garside et al., 2007 & $0-1$ \\
\hline uRTx_CTx & 0.7426 & Garside et al., 2007 & $0-1$ \\
\hline U_TMZ_nodeficit & 0.7314 & Garside et al., 2007 & $0-1$ \\
\hline u_motoricdeficit & 0.381 & Murray et al., 1994* & $0-1$ \\
\hline
\end{tabular}

Utility values (u) for different health states used for the simulation, as well as variation range for probabilistic sensitivity analysis (PSA) for the case of radiotherapy (RTx) and chemotherapy (CTx) with TMZ.

* GBD 1990.

for patients who underwent nTMS and for the control group, as well as the probability of death, were extracted from previously published results from our study group..$^{13}$

\section{Clinical Outcome}

Different clinical outcomes were included in our model in order to differentiate between years of life lost (YLL) and years lived with disability (YLD) due to mild and severe motor deficits. The outcome units were evaluated in quality-adjusted life years (QALYs):

$$
\sum_{i=0}^{1} y(i) * u(i)
$$

$=$ sum of life years spent in the lifespan $i *$ utility during the life period $i$.

Utilities for each stage of treatment were extracted from the Global Burden of Disease Study (GBD 1990), ${ }^{18}$ and from previously published literature $8,10,19,25$ (Table 1). A discount rate of $3 \%$ was applied to health outcomes, as recommended by the WHO CHOICE (CHOosing Interventions that are Cost-Effective) guidelines.

\section{Medical Costs}

Table 2 summarizes the costs included in our evaluation. The costs of each scenario were estimated from a health care system perspective, not including any indirect costs. The costs were estimated according to the following treatment strategy:

- Operation and hospital inpatient stay (with and without performance of nTMS)

- Combined adjuvant therapy: focal RTx (6 weeks, 5 days a week with a fraction of 2 Gy per day) + CTx with an alkylating agent (TMZ $75 \mathrm{mg} / \mathrm{m}^{2}$ per day)

- Adjuvant TMZ therapy after RTx: 1st week $300 \mathrm{mg}$ for 5 days, then 5 cycles of 5 days of $400 \mathrm{mg} /$ dose daily

- In case of tumor recurrence: adjuvant CTx with TMZ: 6 cycles of $400 \mathrm{mg} /$ day for 5 days a week

To calculate the average dosages of TMZ, we assumed a typical patient's body surface of approximately $2 \mathrm{~m}^{2}$, leading to an initial dosage of $150 \mathrm{mg}$ TMZ for the concomitant TMZ therapy.

\section{Model Structure}

We constructed a decision tree-based simulation mod- 
TABLE 2. Unit and total costs

\begin{tabular}{lcclc}
\hline \multicolumn{1}{c}{ Unit } & Cost per Unit (Euros) & Total Cost (Euros) & Description & Variation for PSA \\
\hline cHosp & 600 & $7200-9000$ & Cost of hospital stay & $0 ; 14,400-18,000$ \\
\hline Operation & 4976.21 & 4976.21 & Cost of operation & $0 ; 10,000$ \\
\hline RTx session (Stupp) & 279.70 & 8390.10 & Cost of RTx & $0 ; 16,780$ \\
\hline CTx (mg) (1st half Stupp) & 1.36 & 8568 & Cost of concomitant CTx & $0 ; 17,136$ \\
\hline CTx (mg) (2nd half Stupp) & 1.36 & 15,640 & Cost of 6 cycles TMZ after adjuvant RTx \& CTx & $0 ; 30,000$ \\
\hline nTMS & 842 & 842 & Cost of nTMS & $0 ; 2000$ \\
\hline
\end{tabular}

PSA = probabilistic sensibility analysis.

Costs included in the simulation model, classified by category, unit cost, total cost, and variation range used for sensitivity analysis (PSA).

el, running 1000 microsimulation cycles based on our previously published data from our working group; these previously published data were based on the prospective comparison between resection in patients with and without nTMS ${ }^{13}$ (Fig. 1).

We used repeated random sampling to more accurately reflect our probability distributions. The time horizon included the lifespan of 2 years, therefore opting for the possibility of a second period of CTx with TMZ. Alternative treatment options, such as alkylating agents (carmustine/ lomustine), VEGFR and EGFR inhibitors (bevacizumab/ cetuximab), or other multitargeted drugs, were not included. ${ }^{17}$ A hypothetical cohort, with similar clinical and demographic properties as the patients in our previously published data from prospective analysis (Table 3), was entered in the model. ${ }^{12}$

Prior to surgery, patients would receive either of 2 competing preoperative imaging techniques, dividing the population in 2 groups of interest: one group would receive standard preoperative MRI, which would then be used for intraoperative neuronavigation to localize the motor cortex, while the other group would undergo mapping with nTMS to identify the motor cortex in addition to undergoing the standard preoperative MRI and intraoperative neuronavigation. After the surgical procedure, clinical outcome was assessed and the EOR was measured based on postoperative MRI scans.

We considered 3 different health outcomes (no motor

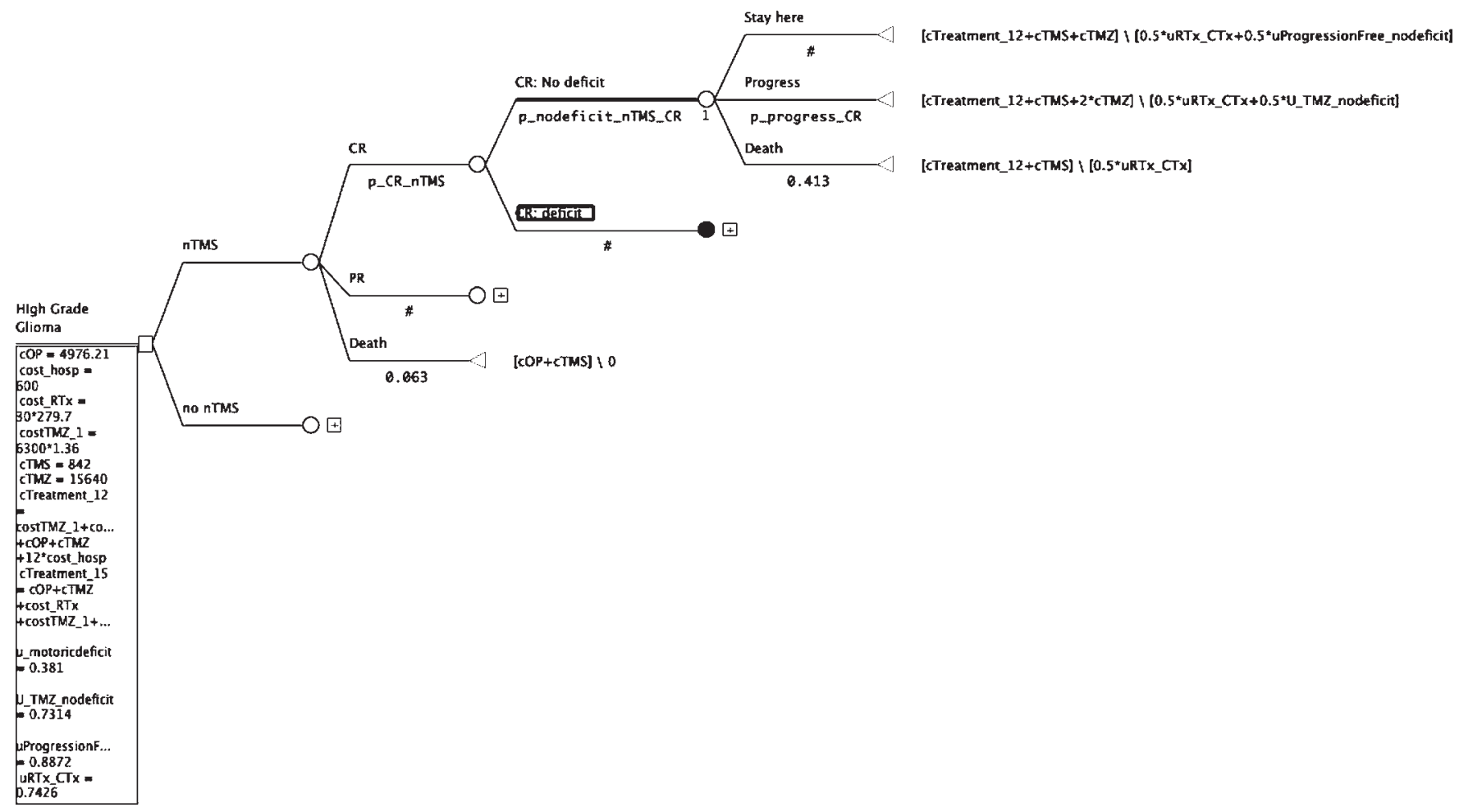

FIG. 1. Basic decision tree displaying 2 treatment options (nTMS vs no nTMS prior to surgery). The transition probability of complete resection (CR) is followed by the health outcome (no motoric deficit vs motoric deficit or death). The last stage includes the possibility of a tumor recurrence. The end node presents the costs and the outcome (utility) of reaching the end point with the specific treatment arm. $c=\operatorname{cost} ; p=$ probability; $u=$ utility. 
TABLE 3. Patient data

\begin{tabular}{lcc}
\hline \multicolumn{1}{c}{ Characteristic } & nTMS & No nTMS \\
\hline Mean age (yrs) & $58 \pm 13.8$ & $60.3 \pm 14.7$ \\
\hline Sex: male (\%) & 64.3 & 64.3 \\
\hline Preop KPS (\%) & 80 & 80 \\
\hline Preop paresis: severe (\%) & 7.1 & 2.9 \\
\hline GTR (\%) & 65.7 & 45.7 \\
\hline New motor paresis (\%) & & \\
\hline After GTR & 17.4 & 28.1 \\
\hline After PR & 12.5 & 34.2 \\
\hline
\end{tabular}

KPS $=$ Karnofsky Performance Status

Patient data and percentage of patients with and without new permanent paresis after GTR and PR according to postoperative MRI, based on Krieg et al., $2015 .{ }^{13}$

deficit, permanent severe paresis [motor deficit $\leq$ grade 3 for the chosen time period], and death) as well as 2 resection rates, GTR and PR, resulting in different clinical outcomes and recurrence risks. From this stage on, each patient can have different clinical courses: progressionfree survival (PFS), tumor recurrence (or progress), and/ or death. These 3 stages were considered end stages, and costs and utilities were analyzed at each of the end branches of our decision tree.

Transition parameters and proportions were again extracted from previously published literature and entered into the model with a specific statistical distribution. ${ }^{8,10,13,19}$

Utilities were estimated and adjusted for adjuvant therapy (RTx and concomitant TMZ) after the operation (uRTx_CTx), recurrent tumor chemotherapy (U_TMZ_nodeficit), a postoperative permanent deficit (u_motoricdefi- cit), and PFS (uProgressionFree_nodeficit). The utilities for each health state are described in Table 1 and estimated from the GBD as well as previously published data comparing cost-efficiencies in intracranial tumor patients.

\section{Transition Probabilities}

In order to account for the uncertainty across our different parameters, we added statistical distributions to the decision tree. Table 4 shows the different transition probabilities and their assigned distributions entered into our model. Transition probabilities, such as the rate of GTR, rate of PR, and clinical outcomes, were assigned to beta distributions, a continuous probability distribution having 2 parameters most commonly used to model one's uncertainty about the probability of success.

The probability of death was confined to a lognormal distribution, and tumor progression/recurrence was assigned to an exponential function. The specific values and their standard deviations, as well as the assigned distributions, were obtained from previously published literature and are described in Table 5.,10,13,25

\section{Probabilistic Sensitivity Analysis}

To consider all occurring uncertainties across our data, and in order to understand and estimate the impact of different variables on our results, we used decision tree probabilities and utilities using random sampling simulation techniques, ${ }^{6,7}$ a stochastic method based on the random sampling of inputs. Estimated variables are repeatedly sampled to determine an empirical distribution for decision-makers. ${ }^{2}$

Sensitivity analysis quantifies and qualifies the uncertainty underlying the decision process for decision-makers in health care policy. ${ }^{1}$ Probabilistic sensitivity analysis

TABLE 4. Probability distributions

\begin{tabular}{lll}
\hline Type & \multicolumn{1}{c}{ Name } & Description \\
\hline Beta & p_GTR_nTMS & Probability of GTR w/nTMS \\
\hline Beta & p_PR_nTMS & Probability of PR w/nTMS \\
\hline Lognormal & p_Death_nTMS & Probability of death w/in 3 mos w/ nTMS \\
\hline Beta & p_GTR_nonTMS & Probability of GTR w/o nTMS \\
\hline Beta & p_PR_nonTMS & Probability of PR w/o nTMS \\
\hline Lognormal & p_death_nonTMS & Probability of death w/in 3 mos w/o nTMS \\
\hline Beta & p_nodeficit_nTMS_GTR & Probability of no deficit w/ GTR \& nTMS \\
\hline Beta & p_deficit_nTMS_PR & Probability of deficit w/ PR \& nTMS \\
\hline Beta & p_deficit_nonTMS_PR & Probability of deficit w/ PR w/o nTMS \\
\hline Beta & p_nodeficit_nonTMS_GTR & Probability no deficit w/ GTR w/o nTMS \\
\hline Beta & p_deficit_nTMS_GTR & Probability of deficit w/ GTR \& nTMS \\
\hline Beta & p_deficit_nonTMS_GTR & Probability of deficit w/ GTR w/o nTMS \\
\hline Beta & p_nodeficit_nTMS_PR & Probability of no deficit w/PR \& nTMS \\
\hline Beta & p_nodeficit_nonTMS_PR & Probability of no deficit w/ PR w/o nTMS \\
\hline Lognormal & p_Death_GTR_nTMS_nodeficit & Probability of death for GTR w/ nTMS \\
\hline Exponential & p_progress_GTR & Probability of tumor recurrence for GTR \\
\hline Exponential & p_progress_PR & Probability of tumor recurrence for PR \\
\hline
\end{tabular}

Description of probability distributions included in our decision model, based on the transition probability extracted from previously published data. 
TABLE 5. Simulation model variables

\begin{tabular}{|c|c|c|c|c|}
\hline Type & Name & Description (probability of) & Value & Source \\
\hline Beta & p_GTR_nTMS & GTR w/ nTMS & 0.6255 & Krieg et al., 2015 \\
\hline Beta & p_PR_nTMS & PR w/nTMS & 0.3115 & Krieg et al., 2015 \\
\hline Lognormal & p_Death_nTMS & Death w/in 3 mos w/ nTMS & 0.063 & Krieg et al., 2015 (2) \\
\hline Beta & p_GTR_nonTMS & GTR w/o nTMS & 0.362 & Krieg et al., 2015 \\
\hline Beta & p_PR_nonTMS & PR w/o nTMS & 0.448 & Krieg et al., 2015 \\
\hline Lognormal & P_death_nonTMS & Death w/in 3 mos w/o nTMS & 0.19 & Krieg et al., 2015 (2) \\
\hline Beta & P_nodeficit_nTMS_GTR & No deficit w/ GTR \& nTMS & 0.871 & Krieg et al., $2015(6)$ \\
\hline Beta & p_nodeficit_nTMS_PR & No deficit w/ PR \& nTMS & 0.867 & Krieg et al., 2015 (6) \\
\hline Beta & P_nodeficit_nonTMS_GTR & No deficit w/ GTR w/o nTMS & 0.833 & Krieg et al., 2015 (6) \\
\hline Beta & P_nodeficit_nonTMS_PR & No deficit w/ PR w/o nTMS & 0.637 & Krieg et al., 2015 (6) \\
\hline Beta & p_deficit_nTMS_GTR & Deficit w/ GTR \& nTMS & 0.129 & Krieg et al., 2015 (6) \\
\hline Beta & P_deficit_nTMS_PR & Deficit w/ PR \& nTMS & 0.133 & Krieg et al., 2015 (6) \\
\hline Exponential & p_progress_GTR & Progress for GTR & 0.0197 & Esteves et al., 2015 (7) \\
\hline Exponential & p_progress_PR & Progress for PR & 0.0086 & Esteves et al., 2015 (7) \\
\hline
\end{tabular}

Distribution values and types included in the simulation model and sources used to extract the data.

(PSA) assigns probability distributions to input values and simulates different scenarios to estimate the overall extent of influence of variables on the overall outcome, such as, in our case, the incremental cost-effectiveness ratio (ICER). Results can be presented in a tornado diagram, listing the different variables by decreasing relative importance for the outcome based on low/median and high values.

The willingness-to-pay (WTP) was set at $3 *$ per capita gross domestic product (GDP) $(35,810.07$ Euros, Germany 2014), following WHO CHOICE (CHOosing Interventions that are Cost-Effective) guidelines for policy makers in health economics. ICER values $<1$ per capita GDP were considered very cost-effective. An ICER value over $3 *$ per capita GDP was above the cost-effectiveness threshold.

\section{Perspective}

The analysis was conducted from a health insurer perspective, aiming to include health benefits with smallest possible growth in costs. It reflects the direct treatment costs and is influenced by the length of hospital stay and rates of complications, such as additional treatments and operations. Indirect costs, such as loss of productivity and further treatment options, such as physiotherapy/ergotherapy/rehabilitation, were not included, and therefore the analysis missed the additional costs of burden of disease after the inpatient period.

\section{Results \\ Base-Case Analysis}

The mean total direct costs per glioma patient, including costs for hospital inpatient days, operation, and adjuvant treatment (Stupp), with and without nTMS were 61,267 and 62,214 Euros, respectively. Further CTx in case of a tumor recurrence added an additional cost of 15,640 Euros (equal to 6 cycles of TMZ). On average, patients who undergo preoperative mapping spent fewer days as hospital inpa- tients compared to the control group without mapping (12 vs 15 days). Table 6 presents the total costs of treatment.

The mean effectiveness amounted to 0.55 QALY, compared to 0.37 QALY without nTMS. This represents an increase of 0.18 QALY. Figure 2 displays the average payoffs for each strategy, obtained by rolling back the decision tree.

\section{Incremental Cost-Effectiveness Ratio}

Table 6 shows the results of 1000 repeated samplings using random sampling simulation. The mean additional cost for nTMS was 7969 Euros; incremental effectiveness amounted to 0.18 QALY. For the average patient, the base-case incremental cost-effectiveness ratio (ICER) was 45,086 Euros/QALY gained (therefore being cost-effective according to the WHO CHOICE guidelines). Considering the indirect costs not included in our analysis, the ICER for a societal perspective would be much lower because of inclusion of the loss of productivity due to motor deficits. Compared to other treatments, nTMS offers a cost-effective preoperative tool for use in treating glioma patients, and should therefore be routinely applied for every patient with a tumor in an eloquent area of the brain.

\section{Uncertainty Analyses}

To determine the uncertainty surrounding cost-effectiveness, we first used 1-way sensitivity analysis.

Figure 3 describes the cost-effectiveness acceptability curve (CEAC), plotting the WTP (x-axis) versus the probability of cost-effectiveness of nTMS. We used a maximum WTP of $3 *$ per capita GDP (= 107,430 Euros) according to WHO CHOICE guidelines. The cost-effectiveness scatterplot shows the uncertainty around our ICER values. Each dot represents 1 cost-effectiveness pair from the simulation results $(\mathrm{n}=1000)$.

We found that our model is particularly sensitive to variations in quality of life during PFS and to the cost of 
TABLE 6. Cost-effectiveness statistics

\begin{tabular}{|c|c|c|}
\hline Parameter & nTMS & no nTMS \\
\hline \multicolumn{3}{|l|}{ Costs (Euros) } \\
\hline Mean & $51,976.56$ & $44,007.10$ \\
\hline SD & 503.82 & 640.63 \\
\hline Minimum & $50,207.07$ & $41,813.87$ \\
\hline $2.5 \%$ & $50,988.93$ & $42,774.97$ \\
\hline $10 \%$ & $51,341.53$ & $43,218.21$ \\
\hline Median & $51,976.90$ & $43,972.95$ \\
\hline $90 \%$ & $52,600.96$ & $44,862.15$ \\
\hline $97.5 \%$ & $52,972.13$ & $45,211.55$ \\
\hline Sum & $51,976,558.70$ & $44,007,099.47$ \\
\hline Size (n) & 1000 & 1000 \\
\hline Variance & $253,832.66$ & $410,405.21$ \\
\hline \multicolumn{3}{|c|}{ Effects (QALY) } \\
\hline Mean & 0.55 & 0.37 \\
\hline SD & 0.02 & 0.02 \\
\hline Minimum & 0.46 & 0.32 \\
\hline $2.5 \%$ & 0.49 & 0.34 \\
\hline $10 \%$ & 0.52 & 0.35 \\
\hline Median & 0.55 & 0.37 \\
\hline $90 \%$ & 0.58 & 0.39 \\
\hline $97.5 \%$ & 0.59 & 0.40 \\
\hline Sum & 550.61 & 373.85 \\
\hline Size (n) & 1000 & 1000 \\
\hline Variance & 0.00 & 0.00 \\
\hline
\end{tabular}

Random sampling simulation cost-effectiveness statistics showing the results of 1000 samplings for subjects with nTMS and without. Mean incremental costs were 7969 Euros, mean incremental effects 0.18 QALY.

TMZ (which is not surprising, as it represents the greatest contribution to overall treatment costs). Other factors, such as cost per day of hospitalization and cost of nTMS, had only little impact on the mean ICER value (Fig. 4).

\section{Perspectives}

Regarding the health-economical perspectives described previously, nTMS provides benefits on each level. From a health care provider perspective, it reduces the length of hospital stay and costs associated with perioperative complications, and it increases the GTR rate.

From a health insurer perspective, as examined in our study, nTMS is comparable to an investment in future health, as it decreases the rate of postoperative paresis and direct treatment costs.

The larger the scale, the higher appears to be the benefit of nTMS: from a societal perspective, the costs saved are increased by the proportion of indirect costs, including rehabilitation, physiotherapy, and productivity loss due to occupational disability.

\section{Discussion \\ General Aspects}

We conducted a cost-effectiveness analysis of imple- mentation of nTMS for preoperative motor mapping versus the gold standard (MRI) in glioma patients. We constructed a decision tree model and used random sampling simulation for dealing with uncertainty around our parameter values. The expected ICER value per gained QALY was around 45,000 Euros, therefore cost-effective and below the WTP following the WHO CHOICE guidelines. Sensitivity analyses suggested a high impact of health utility and costs of TMZ on the expected ICER value.

\section{Special Considerations of the Model}

Since its implementation, nTMS has been increasingly gaining importance in the clinical setting. On average, the incremental costs per TMS patients are only $842 \mathrm{Eu}-$ ros. Meanwhile, the high ICERs result not only from the incremental costs per case, but mainly from the overall survival and therefore additional costs for chemotherapy (main share of total costs). Adding more life years means adding more costs for the overall treatment. If only direct incremental treatment costs are compared (as previously done by Slof et al. for the case of 5-ALA fluorescence, resulting in an ICER of 9021 Euros per QALY ${ }^{21}$ ), the costs of nTMS per patient decrease to 1967 Euros per QALY gained, meaning that it is very cost-effective $(<$ per capita GDP). Esteves et al. included costs of therapy such as chemotherapy and variables such as stable disease and tumor recurrence and found an ICER of 13,000 Euros per QALY gained. ${ }^{8}$ We did not compare nTMS to other additional therapies, as 5-ALA was given to all patients and fMRI is not performed in our department.

In addition to the overestimated costs, the time horizon used (2 years) puts a strong restriction on the possible effects and thereby underestimates the benefits of nTMS. Life years gained, having a high impact on total QALYs gained, are not entirely included in our analysis. We expect that gathering more long-term data and expanding the time horizon to 5 years will decrease the average costs per QALY gained by increasing the effectiveness in terms of overall survival.

A further restriction of our presented model involves the population data used: the input data are provided by previous studies conducted in our department and may not reflect the reality in other clinical departments; thus, we would like to use a broader study population in the future as studies including the nTMS modality improve over time and include more and more patient numbers.

Many factors have not been included in our simulation model. Examples are operation or RTx for recurrent tumors, methylation status, and alternative treatments (carmustine, receptor inhibitors), as well as costs for postoperative physiotherapy, cost of caregivers in case of persistent disability, and the loss of productivity caused by unemployment and YLL (indirect costs).

A highly influential cost factor has been neglected in our model but will be included in further research: HGGs are extremely aggressive tumors, and the vast majority of patients have tumor recurrence despite intensive treatment. The costs of recurrent tumor resections have not been considered, although a prospective study showed that 1 year after diagnosis approximately $15 \%$ of patients with histo- 
V. M. Butenschön et al.
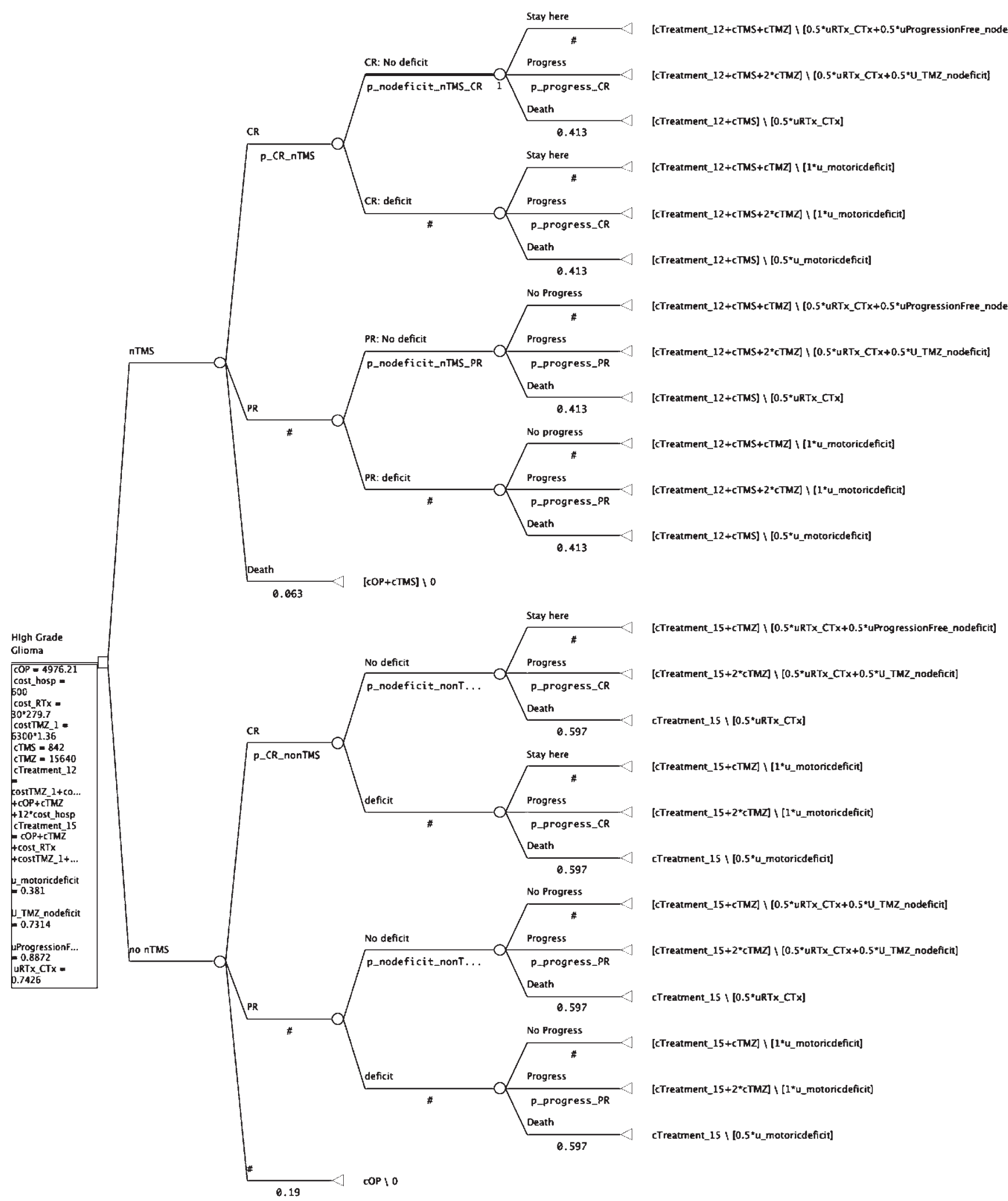

FIG. 2. Costs and effects per selected scenario for both groups: patients undergoing nTMS preoperatively and patients without motoric mapping. nTMS increases the overall effectiveness by 0.18 QALY. 

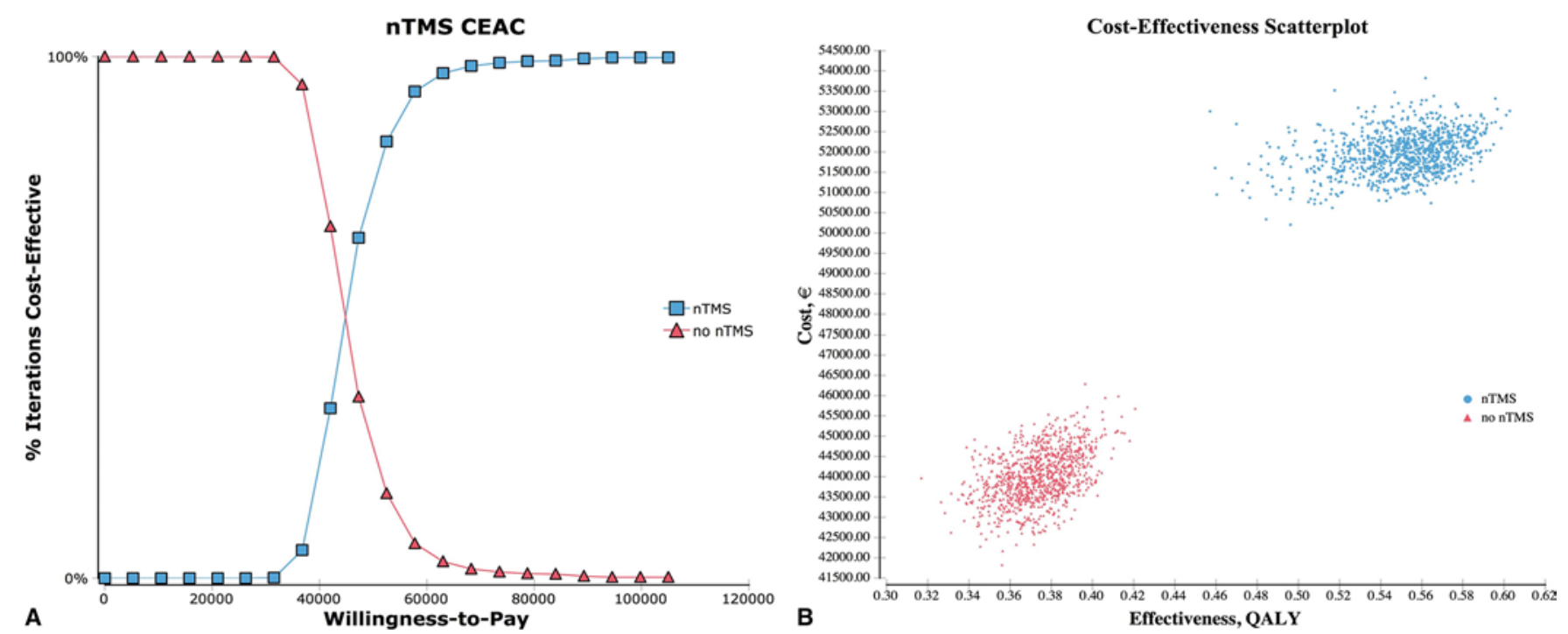

FIG. 3. A: Cost-effectiveness acceptability curve (CEAC) of preoperative nTMS treatment. The willingness-to-pay is set at $3^{*}$ per capita GDP (107,430 Euros). The 2 lines intersect when WTP is equal to the ICER. B: Scatterplot distributions of ICER distribution for 1000 samplings, disclosing the variability within the original study sample.

logically confirmed glioblastoma had undergone resection for recurrence, and at 2 years after diagnosis the rate was approximately $30 \% .^{3}$

In health economics, several perspectives can be adopted while conducting cost-effectiveness analyses. In this study, we used data from a health insurance point of view, accounting for direct treatment costs and health benefits measured in QALYs. If a societal perspective is adopted (maximizing societal health, decreasing overall spending), all occurring costs related to an illness have to be included. Indirect costs in particular play a pivotal role in current economies. A study conducted in Sweden by Blomqvist et al. showed that indirect costs amount to $75 \%$ of the total cost of illness for malignant glioma patients, mainly due to early mortality. ${ }^{4}$ Excluding indirect costs may underestimate the cost-saving aspects of new treatment techniques, such as nTMS.

In this study, we only included HGG patients, and we excluded patients with other tumors, such as metastases, in eloquent areas of the brain. While trying to standardize tumor patients with brain metastases, we face more difficulties caused by differing life expectancies, tumor recurrence probabilities, and systemic chemotherapy treatment, leading to more subgroups than in glioma patients and nonfeasible generalization. Still, by adapting the model to more subtle assumptions, cost-effectiveness analyses could be conducted for patients with all kinds of intracranial tumors.

Sensitivity analyses revealed a high impact of health utilities on the expected ICER. In our case, utility values used were obtained from previously published studies. No prospective investigation of health preference has been conducted, and especially for the case of motoric deficits (corresponding to a utility of 0.381 ), we lack evidencebased utilities properly reflecting health choices. With the increasing use of QALYs in health care decision-making, there is a strong need for more reliable preference weights, and further research needs to focus and to be conducted on health-related utilities. ${ }^{16,24}$

Many indirect beneficial aspects of nTMS have not been included in our analysis. Litofsky et al. reported in 2004 that depression is a common complication after surgical treatment of HGGs. ${ }^{15}$ In addition to the benefits in clinical outcome, nTMS increases patient satisfaction, increasing their understanding of pathology and surgery, and decreasing their anxiety level (Senger et al., unpublished presentation) and may increase their quality of life. Furthermore, a factor not included in our model is the improved quality of life related to a GTR itself. Brown et al. found a significant association between GTR and the experienced health utility after the operation. ${ }^{5}$

Meanwhile, a model is only as good as its assumptions. Most data and units used in this model were prospectively collected but based on nonrandomized studies using historical controls. Working toward more reliable data will provide us the benefit of a more realistic simulation and more precise results.

The increased effectiveness of 0.18 QALY caused by nTMS is, in its outcome, comparable to the effectiveness of 5-ALA (0.16 QALY, reported by Esteves et al. $\left.{ }^{8}\right)$. Furthermore, the sensitivity analysis showed similar results regarding the impact of different variables on the overall outcome: progression-free utility had the highest influence on the ICER value.

As usual, new techniques need to find their way into clinical practice. This study demonstrates that nTMS motor mapping can provide a substantial economical benefit, thereby representing not only an improvement of medical treatment, but also a cost-effective tool for health insurance and society.

\section{Conclusions}

By using a cohort simulation model based on recently 


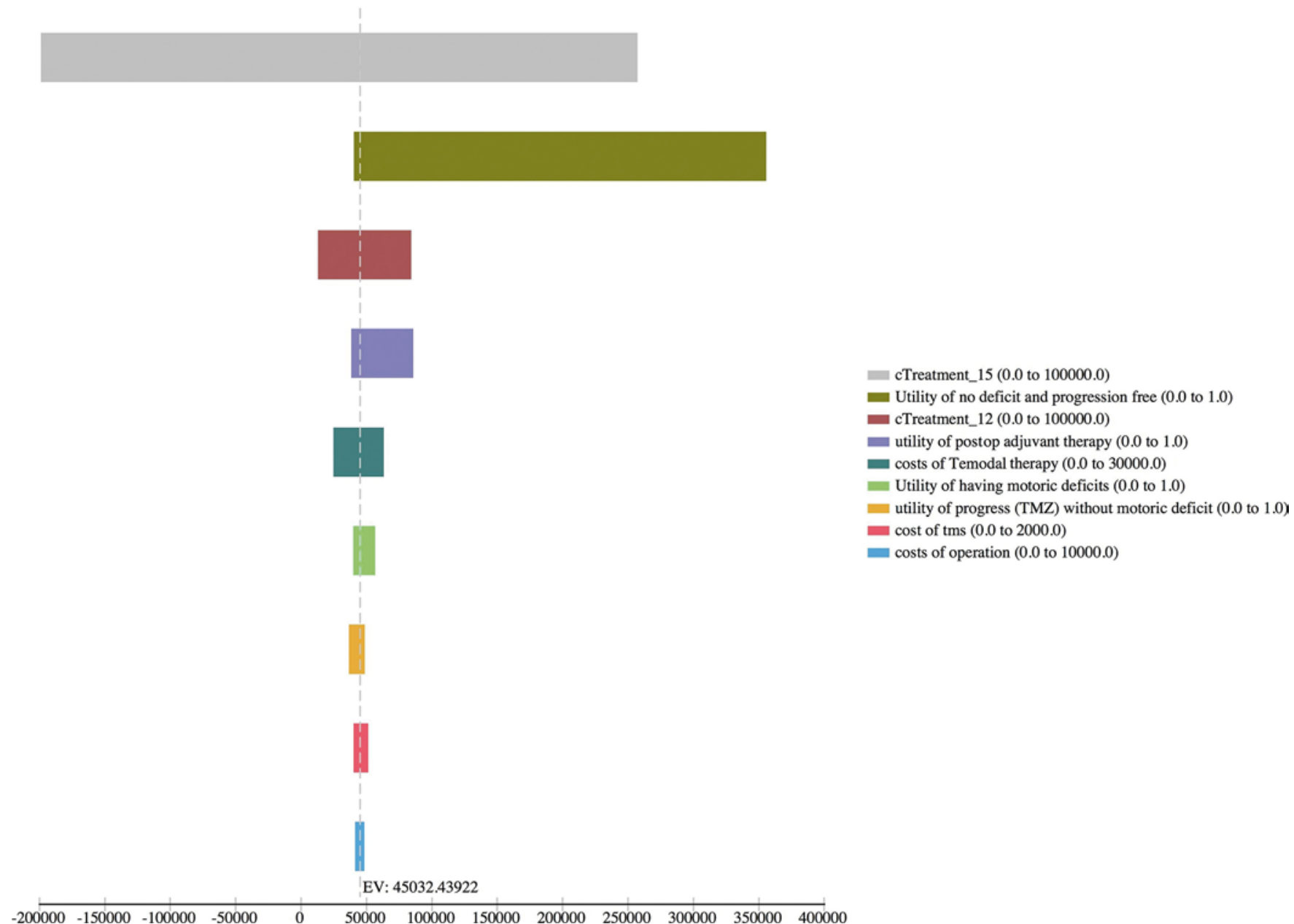

FIG. 4. Tornado diagram presenting the influence of different variables listed by importance. The main impact was achieved by the utility of patients being progression free without any motoric deficit (= their quality of life), while inputs such as cost of nTMS and cost of operation had only little impact on the expected ICER value. EV = expected value.

published data, we evaluated the cost-effectiveness of preoperative motor mapping for HGG patients. For an average patient, the incremental cost of 1 QALY is $45,000 \mathrm{Eu}-$ ros. The ICER is under the WTP threshold described by the WHO CHOICE guidelines and is therefore regarded as cost-effective. Focusing on the incremental costs of nTMS alone without the additional adjuvant treatment, the ICER was even considered "very cost-effective," having a value of approximately 8000 Euros. This economic evaluation supports the significance and clinical relevance of nTMS in a preoperative setting and adds to its clinical benefits a financial sustainability.

Moreover, with this study we provide a model that can be used in the future to analyze new treatment options in neuro-oncology in terms of their general cost-effectiveness.

\section{References}

1. Baio G, Dawid AP: Probabilistic sensitivity analysis in health economics. Stat Methods Med Res 24:615-634, 2015

2. Baltussen RM, Hutubessy RC, Evans DB, Murray CJ: Uncer- tainty in cost-effectiveness analysis. Probabilistic uncertainty analysis and stochastic league tables. Int J Technol Assess

Health Care 18:112-119, 2002

3. Barker FG II, Chang SM, Gutin PH, Malec MK, McDermott MW, Prados MD, et al: Survival and functional status after resection of recurrent glioblastoma multiforme. Neurosurgery 42:709-723, 1998

4. Blomqvist P, Lycke J, Strang P, Törnqvist H, Ekbom A: Brain tumours in Sweden 1996: care and costs. J Neurol Neurosurg Psychiatry 69:792-798, 2000

5. Brown PD, Maurer MJ, Rummans TA, Pollock BE, Ballman $\mathrm{KV}$, Sloan JA, et al: A prospective study of quality of life in adults with newly diagnosed high-grade gliomas: the impact of the extent of resection on quality of life and survival. Neurosurgery 57:495-504, 2005

6. Critchfield GC, Willard KE: Probabilistic analysis of decision trees using Monte Carlo simulation. Med Decis Making 6:85-92, 1986

7. Doubilet P, Begg CB, Weinstein MC, Braun P, McNeil BJ: Probabilistic sensitivity analysis using Monte Carlo simulation. A practical approach. Med Decis Making 5:157-177, 1985

8. Esteves S, Alves M, Castel-Branco M, Stummer W: A pilot cost-effectiveness analysis of treatments in newly diagnosed 
high-grade gliomas: the example of 5-aminolevulinic acid compared with white-light surgery. Neurosurgery 76:552562,2015

9. Frey D, Schilt S, Strack V, Zdunczyk A, Rösler J, Niraula B, et al: Navigated transcranial magnetic stimulation improves the treatment outcome in patients with brain tumors in motor eloquent locations. Neuro Oncol 16:1365-1372, 2014

10. Garside R, Pitt M, Anderson R, Rogers G, Dyer M, Mealing $\mathrm{S}$, et al: The effectiveness and cost-effectiveness of carmustine implants and temozolomide for the treatment of newly diagnosed high-grade glioma: a systematic review and economic evaluation. Health Technol Assess 11:iii-iv, ix-221, 2007

11. Kombos T, Suess O, Brock M: [Cost analysis of intraoperative neurophysiological monitoring (IOM).] Zentralbl Neurochir 63:141-145, 2002 (Ger)

12. Krieg SM, Sabih J, Bulubasova L, Obermueller T, Negwer $\mathrm{C}$, Janssen I, et al: Preoperative motor mapping by navigated transcranial magnetic brain stimulation improves outcome for motor eloquent lesions. Neuro Oncol 16:1274-1282, 2014

13. Krieg SM, Sollmann N, Obermueller T, Sabih J, Bulubas L, Negwer C, et al: Changing the clinical course of glioma patients by preoperative motor mapping with navigated transcranial magnetic brain stimulation. BMC Cancer 15:231, 2015

14. Lacroix M, Abi-Said D, Fourney DR, Gokaslan ZL, Shi W, DeMonte F, et al: A multivariate analysis of 416 patients with glioblastoma multiforme: prognosis, extent of resection, and survival. J Neurosurg 95:190-198, 2001

15. Litofsky NS, Farace E, Anderson F Jr, Meyers CA, Huang W, Laws ER Jr: Depression in patients with high-grade glioma: results of the Glioma Outcomes Project. Neurosurgery 54:358-367, 2004

16. Loomes G, McKenzie L: The use of QALYs in health care decision making. Soc Sci Med 28:299-308, 1989

17. Minniti G, Muni R, Lanzetta G, Marchetti P, Enrici RM: Chemotherapy for glioblastoma: current treatment and future perspectives for cytotoxic and targeted agents. Anticancer Res 29:5171-5184, 2009

18. Murray CJ, Lopez AD, Jamison DT: The global burden of disease in 1990: summary results, sensitivity analysis and future directions. Bull World Health Organ 72:495-509, 1994

19. Rogers G, Garside R, Mealing S, Pitt M, Anderson R, Dyer M, et al: Carmustine implants for the treatment of newly diagnosed high-grade gliomas: a cost-utility analysis. Pharmacoeconomics 26:33-44, 2008

20. Sanai N, Berger MS: Surgical oncology for gliomas: the state of the art. Nat Rev Clin Oncol 15:112-125, 2018

21. Slof J, Díez Valle R, Galván J: Cost-effectiveness of 5-aminolevulinic acid-induced fluorescence in malignant glioma surgery. Neurologia 30:163-168, 2015

22. Stummer W, Reulen HJ, Meinel T, Pichlmeier U, Schumacher W, Tonn JC, et al: Extent of resection and survival in glioblastoma multiforme: identification of and adjustment for bias. Neurosurgery 62:564-576, 2008

23. Tarapore PE, Tate MC, Findlay AM, Honma SM, Mizuiri D, Berger MS, et al: Preoperative multimodal motor mapping: a comparison of magnetoencephalography imaging, navigated transcranial magnetic stimulation, and direct cortical stimulation. J Neurosurg 117:354-362, 2012

24. Whitehead SJ, Ali S: Health outcomes in economic evaluation: the QALY and utilities. Br Med Bull 96:5-21, 2010

25. Wu B, Miao Y, Bai Y, Ye M, Xu Y, Chen H, et al: Subgroup economic analysis for glioblastoma in a health resourcelimited setting. PLoS One 7:e34588, 2012

\section{Disclosures}

BM and SMK report being consultants for Brainlab AG (Munich, Germany). SMK reports being a consultant for Nexstim Plc. (Helsinki, Finland).

\section{Author Contributions}

Conception and design: Krieg, Butenschön. Acquisition of data: Krieg, Butenschön, Ille, Sollmann. Analysis and interpretation of data: Krieg, Butenschön. Drafting the article: Krieg, Butenschön. Critically revising the article: Krieg, Ille, Sollmann. Reviewed submitted version of manuscript: Krieg, Ille, Sollmann, Meyer. Approved the final version of the manuscript on behalf of all authors: Krieg. Statistical analysis: Butenschön. Administrative/ technical/material support: Krieg, Meyer. Study supervision: Krieg, Meyer.

\section{Correspondence}

Sandro M. Krieg: Technische Universität München, Klinikum rechts der Isar, Munich, Germany. sandro.krieg@tum.de. 